\title{
Scattering and Bound States of a Spin-1/2 Neutral Particle in the Cosmic String Spacetime
}

\author{
Fabiano M. Andrade, ${ }^{1,2}$ Cleverson Filgueiras, ${ }^{3}$ and Edilberto O. Silva ${ }^{4}$ \\ ${ }^{1}$ Department of Computer Science and Department of Physics and Astronomy, University College London, London WC1E 6BT, UK \\ ${ }^{2}$ Departamento de Matemática e Estatística, Universidade Estadual de Ponta Grossa, 84030-900 Ponta Grossa, PR, Brazil \\ ${ }^{3}$ Departamento de Física, Universidade Federal de Lavras, Caixa Postal 3037, 37200-000 Lavras, MG, Brazil \\ ${ }^{4}$ Departamento de Física, Universidade Federal do Maranhão, 65085-580 São Luís, MA, Brazil
}

Correspondence should be addressed to Fabiano M. Andrade; fmandrade@uepg.br

Received 11 November 2016; Accepted 11 January 2017; Published 31 January 2017

Academic Editor: Andrzej Okniński

Copyright ( 2017 Fabiano M. Andrade et al. This is an open access article distributed under the Creative Commons Attribution License, which permits unrestricted use, distribution, and reproduction in any medium, provided the original work is properly cited. The publication of this article was funded by $\mathrm{SCOAP}^{3}$.

In this paper the relativistic quantum dynamics of a spin-1/2 neutral particle with a magnetic moment $\mu$ in the cosmic string spacetime is reexamined by applying the von Neumann theory of self-adjoint extensions. Contrary to previous studies where the interaction between the spin and the line of charge is neglected, here we consider its effects. This interaction gives rise to a point interaction: $\nabla \cdot \mathbf{E}=(2 \lambda / \alpha) \delta(r) / r$. Due to the presence of the Dirac delta function, by applying an appropriated boundary condition provided by the theory of self-adjoint extensions, irregular solutions for the Hamiltonian are allowed. We address the scattering problem obtaining the phase shift, $S$-matrix, and the scattering amplitude. The scattering amplitude obtained shows a dependency with energy which stems from the fact that the helicity is not conserved in this system. Examining the poles of the $S$-matrix we obtain an expression for the bound states. The presence of bound states for this system has not been discussed before in the literature.

\section{Introduction}

Theory of topological defects is a natural framework for studying properties of physical systems. In cosmology, the origin of defects can be understood as a sequence of phase transitions in the early universe. These processes occur with critical temperatures which are related to the corresponding symmetry spontaneously breaking scales [1-3]. These phase transitions can give rise to topologically stable defects, for example, domain walls, strings, and monopoles [4]. Topological defects are also found in condensed matter systems. In these systems, they appears as vortices in superconductors, domain wall in magnetic materials, and dislocations of crystalline substances, among others. An important property that can be verified in topological defects is that they are described by a spacetime metric with a Riemann-Christoffel curvature tensor which is null everywhere except on the defects. Here, we look for a cosmic string, which is a linear topological defect with a conical singularity at the origin. The interest in this subject has contributed to the understanding and advancement of other physical phenomena occurring in the universe and also in the context of nonrelativistic physics. For example, in the galaxy formation $[5,6]$, to study vortex solutions in nonabelian gauge theories with spontaneous symmetry breaking [7] and to study the gravitational analogue of the Aharonov-Bohm effect [8-12]. In recent developments, cosmic strings have been considered to analyze solutions in de Sitter and anti-de Sitter spacetimes [13], to study the thermodynamic properties of a neutral particle in a magnetic cosmic string background by using an approach based on the partition function method [14], to compute the vacuum polarization energy of string configurations in models similar to the standard model of particle physics [15], to find the deflection angle in the weak limit approximation by a spinning cosmic string in the context of the EinsteinCartan theory of gravity [16], to analyze numerically the behavior of the solutions corresponding to an Abelian string in the framework of the Starobinsky model [17], to study 
solutions of black holes [18], to investigate the average rate of change of energy for a static atom immersed in a thermal bath of electromagnetic radiation [19], to study Hawking radiation of massless and massive charged particles [20], to study the non-Abelian Higgs model coupled with gravity [21], in the quantum dynamics of scalar bosons [22], hydrodynamics [23], to study the nonrelativistic motion of a quantum particle subjected to magnetic field [24], to investigate dynamical solutions in the context of super-critical tensions [25] and Higgs condensate [26], to analyze the effects on spin current and Hall electric field [27, 28], to investigate the dynamics of the Dirac oscillator $[29,30]$, to study noninertial effects on the ground state energy of a massive scalar field [31] and Landau quantization [32], and to investigate the quantum vacuum interaction energy [33].

In the present work, we study the quantum dynamics of a spin-1/2 neutral particle in the presence of an electric field due to an infinitely long, infinitesimally thin line of charge along the $z$-axis of the cosmic string, with constant charge density on it. This model has been studied in [34] in the nonrelativistic regime and, for this particular case, only the scattering problem was considered. The present system is an adaptation of the usual Aharonov-Casher problem [35] (which is dual to the Aharonov-Bohm problem [36]), where now effects of localized curvature are included in the model. We reexamine this problem by using the von Neumann theory of self-adjoint extensions [37, 38]. We address the relativistic case and investigate some questions that were not considered in the previous studies, as, for example, the existence of bound states. For this, we solve the scattering problem and derive the $S$-matrix in order to obtain such bound states.

The plan of this work is the following. In Section 2, we derive the Dirac-Pauli equation in the cosmic string spacetime without neglecting the term which depends explicitly on the spin. Arguments based on the theory of self-adjoint extension are given in order to make clear the reasons why we should consider the spin effects in the dynamics of the system. In Section 3, we study the Dirac-Pauli Hamiltonian via the von Neumann theory of self-adjoint extension. We address the scattering scenario within the framework of Dirac-Pauli equation. Expressions for the phase shift, $S$-matrix, and bound states are derived. We also make an investigation on the helicity conservation problem in the present framework. A brief conclusion is outlined in Section 4 .

\section{The Relativistic Equation of Motion}

The model that we address here consists of a spin-1/2 neutral particle with mass $M$ and magnetic moment $\mu$, moving in an external electromagnetic field $F_{\mu \nu}$ in the cosmic string spacetime, described by the line element in cylindrical coordinates,

$$
d s^{2}=c^{2} d t^{2}-d r^{2}-\alpha^{2} r^{2} d \varphi^{2}-d z^{2},
$$

with $-\infty<(t, z)<\infty, r \geq 0$, and $0 \leq \varphi \leq 2 \pi$ and $\alpha$ is given in terms of the linear mass density $\widetilde{m}$ of the cosmic string by $\alpha=1-4 \widetilde{m} / c^{2}$. This metric has a cone-like singularity at $r=0$ [39]. In this system, the fermion particle is described by a four-component spinorial wave function $\Psi$ obeying the generalized Dirac-Pauli equation in a nonflat spacetime, which should include the spin connection in the differential operator. Moreover, in order to make the DiracPauli equation valid in curved spacetime, we must rewrite the standard Dirac matrices, which are written in terms of the local coordinates in the Minkowski spacetime, in terms of global coordinates. This can be accomplished by using the inverse vierbeins $e_{\bar{a}}^{\mu}$ through the relation $\gamma^{\mu}=e_{\bar{a}}^{\mu} \gamma^{\bar{a}}(\mu, \bar{a}=$ $0,1,2,3)$, with $\gamma^{\bar{a}}=\left(\gamma^{\overline{0}}, \gamma^{\bar{i}}\right)$ being the standard gamma matrices. The equation of motion governing the dynamics of this system is the modified Dirac-Pauli equation in the curved space

$$
\left[i \hbar \gamma^{\mu}\left(\partial_{\mu}+\Gamma_{\mu}\right)-\frac{\mu}{2 c} \sigma^{\mu \nu} F_{\mu \nu}-M c\right] \Psi=0
$$

with $\sigma^{\mu \nu}=i\left[\gamma^{\mu}, \gamma^{\nu}\right] / 2,\left(F_{0 i}, F_{i j}\right)=\left(E^{i}, \epsilon_{i j k} B^{k}\right)$, and $\left(\sigma^{0 j}, \sigma^{i j}\right)=$ $\left(i \alpha^{j},-\epsilon_{i j k} \Sigma^{k}\right)$, where $E^{i}$ and $B^{k}$ are the electric and magnetic field strengths and $\Sigma^{k}$ is the spin operator. Here, we use the same vierbein of [40], where the spinorial affine connection $\Gamma_{\mu}$ has been calculated in detail. Moreover, in this work, we are only interested in the planar dynamics of a spin-1/2 neutral particle under the action of a radial electric field. In this manner we require that $p_{z}=z=0$ and $B^{k}=0$ for $k=1,2,3$. Furthermore, according to the tetrad postulated [41], the matrices $\gamma^{\bar{a}}$ can be any set of constant Dirac matrices in a such way that we are free to choose a representation for them. We choose to work in a representation in which the Dirac matrices are given in terms of the Pauli matrices, namely $[42,43]$,

$$
\begin{aligned}
\beta & =\gamma^{\overline{0}}=\sigma^{3}, \\
\gamma^{\overline{1}} & =i \sigma^{2}, \\
\gamma^{\overline{2}} & =-i s \sigma^{1},
\end{aligned}
$$

where $\left(\sigma^{1}, \sigma^{2}, \sigma^{3}\right)$ are the Pauli matrices and $s$ is twice the spin value, with $s=+1$ for spin "up" and $s=-1$ for spin "down." In this representation, the only nonvanishing component of the spinorial affine connection $\Gamma_{\mu}$ is found to be

$$
\Gamma_{\varphi}=-i \frac{(1-\alpha)}{2} s \sigma^{z}
$$

For the field configuration, we consider the electric field due to a linear charge distribution, superposed to the cosmic string. The expression for this field seems to be

$$
E_{r}=\frac{2 \lambda}{\alpha r}
$$

Therefore, the second-order equation associated with (2) reads

$$
\widehat{H} \Phi=k^{2} \Phi
$$


with

$$
\begin{aligned}
\widehat{H}= & -\nabla_{\alpha}^{2}-\frac{(1-\alpha) s \sigma^{z}}{i \alpha^{2} r^{2}} \partial_{\varphi}+\frac{(1-\alpha)^{2}}{4 \alpha^{2} r^{2}}+\frac{2 \mu s}{\hbar c} \frac{E_{r}}{i \alpha r} \partial_{\varphi} \\
& +\frac{\mu}{\hbar c}\left(\partial_{r} E_{r}\right) \sigma^{z}-\frac{\mu}{\hbar c} \frac{(1-\alpha) E_{r}}{\alpha r} \sigma^{z}+\frac{\mu^{2}}{\hbar^{2} c^{2}} E_{r}^{2},
\end{aligned}
$$

where $\nabla_{\alpha}^{2}=\partial_{r}^{2}+(1 / r) \partial_{r}+\left(1 / \alpha^{2} r^{2}\right) \partial_{\varphi}^{2}$ is the Laplace-Beltrami operator in the conical space and $k^{2}=\left(\mathscr{E}^{2}-M^{2} c^{4}\right) / \hbar^{2} c^{2}$. As the angular momentum $\widehat{J}=-i \partial_{\varphi}+(s / 2) \sigma^{z}$ commutes with the $\widehat{H}$, it is possible to decompose the fermion field as

$$
\Phi=\left(\begin{array}{l}
\psi \\
\chi
\end{array}\right)=\left(\begin{array}{c}
\sum_{m} f_{m}(r) e^{i m \varphi} \\
\sum_{m} g_{m}(r) e^{i(m+s) \varphi}
\end{array}\right),
$$

where $m=0, \pm 1, \pm 2, \pm 3, \ldots$ is the angular momentum quantum number. In this manner, the radial equation for $f_{m}(r)$ is

$$
h f_{m}(r)=k^{2} f_{m}(r)
$$

with

$$
\begin{aligned}
& h=h_{0}+\frac{\eta}{\alpha} \frac{\delta(r)}{r}, \\
& h_{0}=-\frac{d^{2}}{d r^{2}}-\frac{1}{r} \frac{d}{d r}+\frac{j^{2}}{r^{2}},
\end{aligned}
$$

where

$$
j=\frac{m+s \eta}{\alpha}-\frac{s(1-\alpha)}{2 \alpha}
$$

is the effective angular momentum and

$$
\eta=\frac{\phi}{\phi_{0}} .
$$

Here, $\phi=4 \pi \lambda$ is the electric flux of the electric field and $\phi_{0}=$ $h c / \mu$ is the quantum of electric flux.

As far as we know, only the scattering problem for the Hamiltonian in (10) has been studied in [34]. However, there, the spin effect was not taken into account once the author imposed the regularity of the wave function at the origin. The inclusion of spin gives rise to the Dirac delta function potential, which comes from the interaction between the spin and the line of charge, and its inclusion has effects on the scattering phase shift, giving rise to an additional scattering phase shift [44]. Thus, the main aim of this work is to show that there are bound states due to the presence of the Dirac delta function. The approach adopted here is that of the self-adjoint extensions [38], which has been used to deal with singular Hamiltonians, for instance, in the study of spin-1/2 Aharonov-Bohm system and cosmic strings [45, 46], in the Aharonov-Bohm-Coulomb problem [47-50] and in the equivalence between the self-adjoint extension and normalization [51].

\section{Scattering and Bound States Analysis}

In this section, we obtain the $S$-matrix and from its poles an expression for the bound states is obtained. Before we solve (9), let us first analyze the Hamiltonian $h_{0}$.

In the von Neumann theory of self-adjoint extensions, a Hermitian operator $\widehat{O}\left(\widehat{O}=\widehat{O}^{\dagger}\right)$ defined in a dense subset of a Hilbert space has deficiency indices $\left(n_{+}, n_{-}\right)$, which are the sizes of the deficiency subspaces spanned by the solutions for

$$
\widehat{O} \chi_{ \pm}= \pm i \chi_{ \pm} \text {. }
$$

When the dimension of the deficiency subspace is zero, the operator is self-adjoint and it has no additional self-adjoint extension. When the dimension of the deficiency spaces is not zero the operator is not self-adjoint. If $n_{+}=n_{-}=n$ the operator admits a self-adjoint extension parametrized by $n \times n$ unitary matrix. However, if the deficiency indices are not equal, the operator has no self-adjoint extensions. By standard results, it is well-known that the Hamiltonian $h_{0}$ has deficiency indices $(1,1)$ and it is self-adjoint for $|j| \geq 1$, whereas for $|j|<1$ it is not self-adjoint and admits a oneparameter family of self-adjoint extensions [52]. Actually, $h$ can be interpreted as a self-adjoint extension of $h_{0}$ [53]. All the self-adjoint extensions of $h_{0}, h_{0, v}$, are accomplished by requiring the boundary condition at the origin [37]

$$
v f_{0, j}=f_{1, j},
$$

where $-\infty<v \leq \infty$ and $-1<j<1$. The boundary values are

$$
\begin{aligned}
& f_{0, j}=\lim _{r \rightarrow 0^{+}} r^{|j|} f_{m}(r), \\
& f_{1, j}=\lim _{r \rightarrow 0^{+}} \frac{1}{r^{|j|}}\left[f_{m}(r)-f_{0, j} \frac{1}{r^{|j|}}\right] .
\end{aligned}
$$

In (15) $v$ is the self-adjoint extension parameter. It turns out that $1 / v$ represents the scattering length of $h_{0, v}$ [38]. For $v=\infty$ (the Friedrichs extension of $h_{0}$ ), one has the free Hamiltonian (without spin) with regular wave functions at the origin $\left(f_{m}(0)=0\right)$. This situation is equivalent to impose the Dirichlet boundary condition on the wave function. On the other hand, if $|\nu|<\infty, h_{0, \nu}$ describes a point interaction at the origin. In this latter case the boundary condition permits $r^{-|j|}$ singularity in the wave functions at the origin [54].

Let us now discuss for which values of the angular moment quantum number $m$, the operator $h_{0}$ is not selfadjoint. In fact, these values depend on the variables $\alpha$ and $\eta$. As discussed in [34], $0<\alpha<1$ represents a positive curvature and a planar deficit angle, corresponding to a conical spacetime. On the other hand, $\alpha>1$ represents a negative curvature and an excess of planar angle, corresponding to an anticonical spacetime. Finally, $\alpha=1$ corresponds to a flat space. Then, we focus on a conical spacetime. For the electric flux $\eta$ let us adopt the decomposition defined by [55]

$$
\eta=N+\beta,
$$

where $N$ is an integer and

$$
0 \leq \beta<1 .
$$




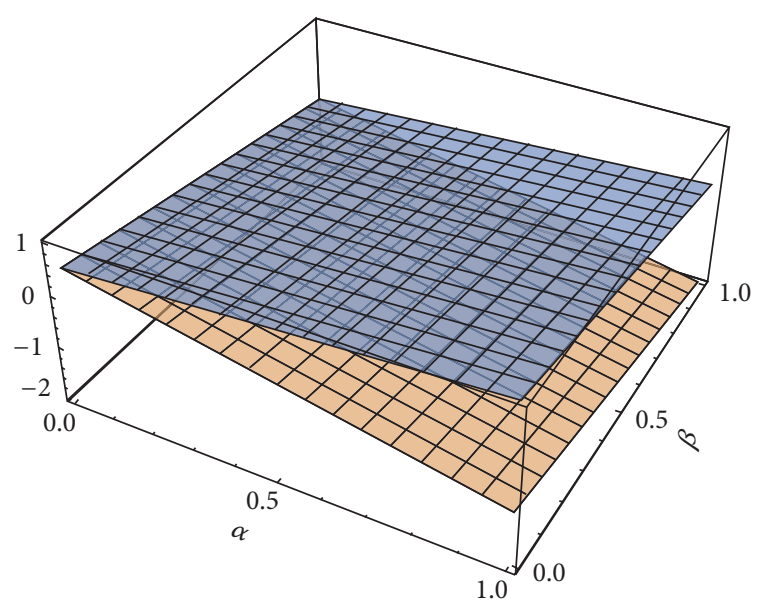

FIGURE 1: In this figure we plot the planes $\pi_{-}(\alpha, \beta)$ (orange, bottom) and $\pi_{+}(\alpha, \beta)$ (blue, top) for $N=0$ and $s=+1$. The region between the two planes is that in which the operator $h_{0}$ is not self-adjoint.

The inequality $|j|<1$ then reads

$$
\pi_{-}(\alpha, \beta)<m<\pi_{+}(\alpha, \beta),
$$

with $\pi_{ \pm}(\alpha, \beta)= \pm \alpha-[(2 \beta+\alpha-1) s / 2-s N]$. In Figure 1 we plot the planes $\pi_{ \pm}(\alpha, \beta)$ for $N=0$ and $s=+1$. The region between these two planes is that in which the operator $h_{0}$ is not selfadjoint. In Figure 2 we show cross sections of this region for some particular values of the deficit angle $\alpha$. We can observe in Figure 2(a) that, for $\alpha=0.25$, only for $m=0$ the operator $h_{0}$ is not self-adjoint, whereas for $\alpha=0.50$ (Figure 2(b)) the operator $h_{0}$ is not self-adjoint for $m=0$ and $m=-1$, but not for both values of $m$ at the same time for the whole range of $\beta$ values. Indeed, a necessary condition for the operator $h_{0}$ not being self-adjoint for the state with $m=-1$ is $\alpha>1-2 \beta / 3$. In fact, this condition is also valid for $N \neq 0$ and, in this latter case, the $m$ values for which $h_{0}$ is not self-adjoint are shifted to the values $s N$ and $s N-1$. For $\alpha=0.75$ (Figure 2(c)) we can observe that there is a range of the values of $\beta$ in which, for both values of $m=0$ and $m=-1$, the operator $h_{0}$ is not self-adjoint. And last but not least, $\alpha=1.0$ (see Figure 2(d)) is the only situation in which the operator $h_{0}$ is not self-adjoint for both values of angular momentum quantum number for the whole range of $\beta$ (the unique exception is $\beta=0$ ).

Now, let us come back to the solution of (9). As a matter of fact, it is the Bessel differential equation. Thus, the general solution for $r \neq 0$ is seen to be

$$
f_{m}(r)=a_{m} J_{|j|}(k r)+b_{m} J_{-|j|}(k r),
$$

where $J_{\nu}(z)$ is the Bessel function of fractional order. The coefficients $a_{m}$ and $b_{m}$ represent the contributions of the regular and irregular solutions at the origin, respectively. Thus making use of the boundary condition in (15) in the subspace $|j|<1$, a relation between the coefficients is obtained, namely,

$$
b_{m}=-\mu_{\nu} a_{m}
$$

where the term $\mu_{\nu}$ is given by

$$
\mu_{v}=\frac{k^{2|j|} \Gamma(1-|j|) \sin (|j| \pi)}{4^{|j|} \Gamma(1+|j|) \nu+k^{2|j|} \Gamma(1-|j|) \cos (|j| \pi)},
$$

where $\Gamma(z)$ is the gamma function. Therefore, in this subspace the solution reads

$$
f_{m}(r)=a_{m}\left[J_{|j|}(k r)-\mu_{\nu} J_{-|j|}(k r)\right] .
$$

The above equation shows that the self-adjoint extension parameter $v$ controls the contribution of the irregular solution $J_{-|j|}$ for the wave function. As a result, for $v=\infty$, we have $\mu_{\infty}=0$, and there is no contribution of the irregular solution at the origin for the wave function. Consequently, the total wave function reads

$$
\psi=\sum_{m=-\infty}^{\infty} a_{m} J_{|j|}(k r) e^{i m \varphi}
$$

It is well-known that the coefficient $a_{m}$ must be chosen in a such way that $\psi$ represents a plane wave that is incident from the right. In this manner, we obtain the result

$$
a_{m}=e^{-i|j| \pi / 2} .
$$

The scattering phase shift can be obtained from the asymptotic behavior of (24). This leads to

$$
\delta_{m}=\frac{\pi}{2}(|m|-|j|) .
$$

This is the scattering phase shift of the Aharonov-Casher effect in the cosmic string background. It is worthwhile to note that, for $\alpha=1$, it reduces to the phase shift for the usual Aharonov-Casher effect in flat space $\delta_{m}=\pi(|m|-|m+s \eta|) / 2$ [35].

On the other hand, for $|\nu|<\infty$, the contribution of the irregular solution modifies the scattering phase shift to

$$
\delta_{m}^{v}=\delta_{m}+\arctan \left(\mu_{\nu}\right) .
$$

Thus one obtains

$$
S_{m}^{\nu}=e^{2 i \delta_{m}^{\nu}}=e^{2 i \delta_{m}}\left(\frac{1+i \mu_{\nu}}{1-i \mu_{\nu}}\right),
$$

which is the expression for the $S$-matrix in terms of the phase shift. As a result, one observes that in this latter case there is an additional scattering for any value of the selfadjoint extension parameter $v$. When $v=\infty$, we have the $S$-matrix for the Aharonov-Casher effect on the cosmic string background, as it should be.

The $S$-matrix or scattering matrix relates incoming and outgoing wave functions of a physical system undergoing a scattering process. Bound states are identified as the poles of the $S$-matrix in the upper half in the complex $k$ plane. In this manner, the poles are determined at the zeros of the denominator in (28) with the replacement $k \rightarrow i \kappa$ with $\kappa=\sqrt{-\left(\mathscr{E}^{2}-M^{2} c^{4}\right) / \hbar^{2} c^{2}}$. Therefore, for $v<0$, one can 


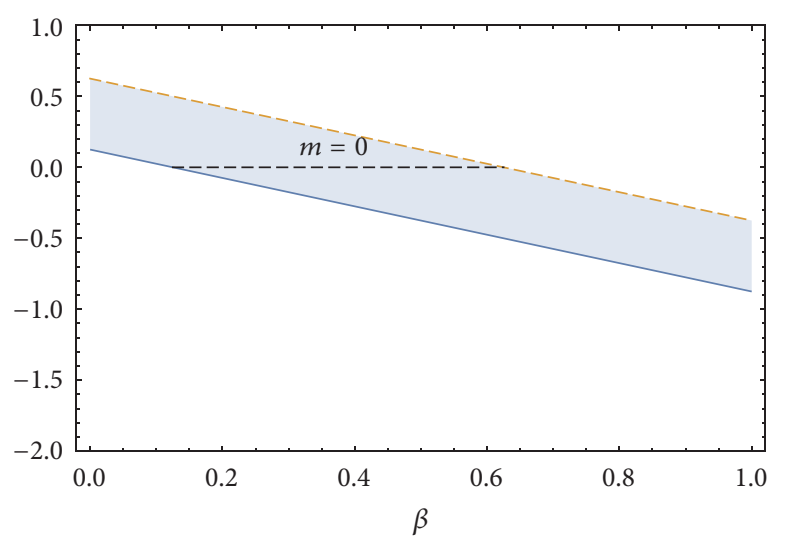

(a)

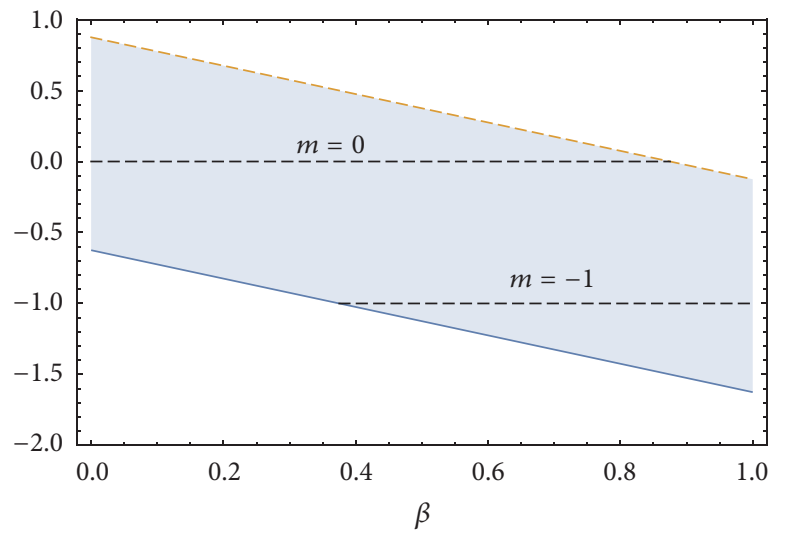

(c)

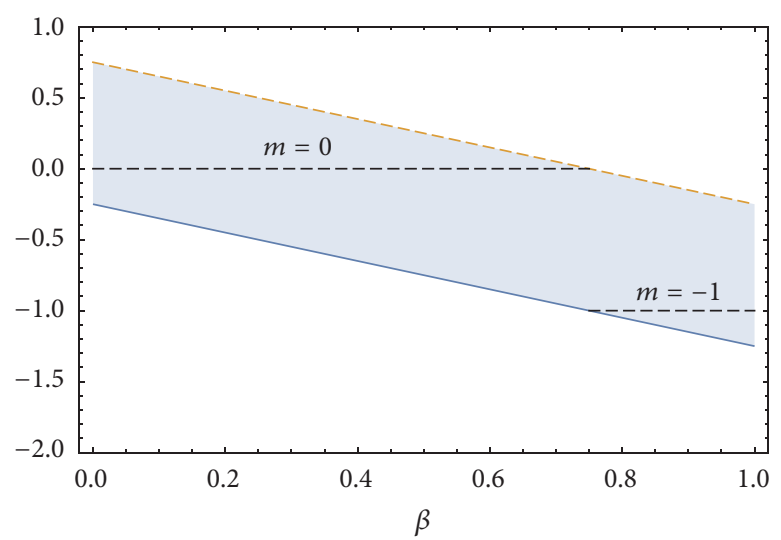

(b)

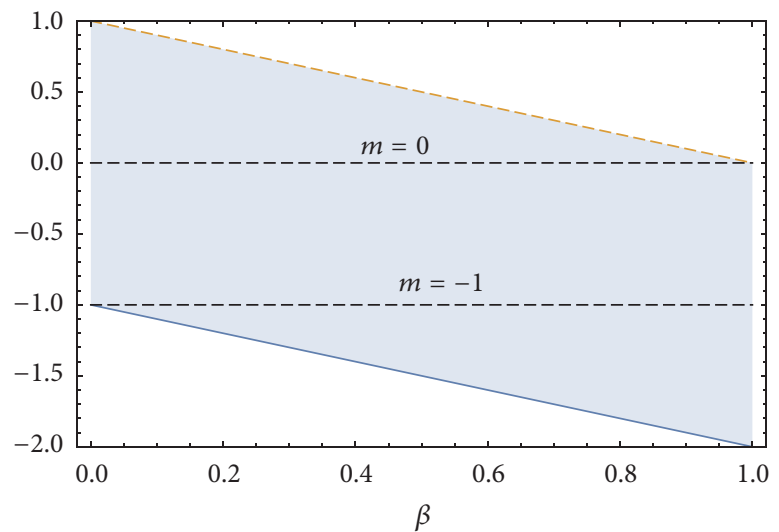

(d)

Figure 2: Cross sections of Figure 1 for different values of the deficit angle: (a) $\alpha=0.25$, (b) $\alpha=0.50$, (c) $\alpha=0.75$, and (d) $\alpha=1.00$. The shaded area schematically represents that area in which the operator $h_{0}$ is not self-adjoint. The dashed lines represent the values of angular moment quantum number.

determine that the present system has a bound state with energy given

$$
\mathscr{E}= \pm \sqrt{M^{2} c^{4}-4 \hbar^{2} c^{2}\left[-v \frac{\Gamma(1+|j|)}{\Gamma(1-|j|)}\right]^{1 /|j|}}
$$

and the normalized radial bound state wave function is

$$
\begin{aligned}
\left(\begin{array}{c}
f_{m}(r) \\
g_{m}(r)
\end{array}\right)= & \sqrt{\frac{2 \alpha \kappa^{2} / \pi}{|j| \alpha \csc (|j| \pi)+\left|j^{\prime}\right| \csc \left(\left|j^{\prime}\right| \pi\right)}} \\
& \times\left(\begin{array}{c}
K_{|j|}(\kappa r) \\
K_{\left|j^{\prime}\right|}(\kappa r)
\end{array}\right),
\end{aligned}
$$

where $j^{\prime}=j+(s / \alpha)(2-\alpha)$ and $K_{\nu}(z)$ is the modified Bessel function of the second kind. So, there are bound states when the self-adjoint extension parameter is negative. In the nonrelativistic limit and for $\alpha=1$, (29) coincides with the bound state energy found in [56] for the Aharonov-Casher effect in the flat space.
As a result, it is possible to express the $S$-matrix in terms of the bound state energy. The result seems to be

$$
S_{m}^{\nu}=e^{2 i \delta_{m}}\left[\frac{e^{2 i \pi|j|}-(\kappa / k)^{2|j|}}{1-(\kappa / k)^{2|j|}}\right] .
$$

Once we have obtained the $S$-matrix, it is possible to write down the scattering amplitude $f(k, \varphi)$. The result is

$$
\begin{aligned}
& f(k, \varphi)=\frac{1}{\sqrt{2 \pi i k}} \sum_{m=-\infty}^{\infty}\left(S_{m}^{v}-1\right) e^{i m \varphi} \\
& =\frac{1}{\sqrt{2 \pi i k}}\left\{\sum_{m \in\{|j| \geq 1\}}\left(e^{2 i \delta_{m}}-1\right) e^{i m \varphi}\right. \\
& \left.+\sum_{m \in\{|j|<1\}}\left[e^{2 i \delta_{m}}\left(\frac{1+i \mu_{\nu}}{1-i \mu_{\nu}}\right)-1\right] e^{i m \varphi}\right\} .
\end{aligned}
$$

In scattering problems the length scale is set by $1 / k$; thus the scattering amplitude $f(k, \varphi)$ would be a function of angle alone, multiplied by $1 / k$ [57]. However, we observe that $f(k, \varphi)$ has a dependence on $\mu_{v}$, which in its turn has explicit 
dependence on $k$ (see (22)). This behavior is associated with the failure of helicity conservation. The helicity operator, defined by

$$
\widehat{h}=\boldsymbol{\Sigma} \cdot\left(-i \nabla_{\alpha}-e \mathbf{A}\right),
$$

obeys the equation

$$
\frac{d \widehat{h}}{d t}=e \boldsymbol{\Sigma} \cdot \mathbf{E}
$$

where $\boldsymbol{\Sigma}$ is the spin operator and in (33) $\mathbf{A}$ is the potential vector, which is absent in the present problem. Therefore, due to the presence of electric field the helicity is not conserved.

\section{Conclusions}

In this work, we reexamined the relativistic quantum dynamics of a spin-1/2 neutral particle in the cosmic string spacetime. This problem has been studied in [34] in the nonrelativistic scenario. However only the scattering solutions were studied and without taking into account the possibility of bound states. Here, we have showed that the inclusion of electron spin, which gives rise to a point interaction, changes the scattering phase shift and consequently the $S$-matrix. The results were obtained by imposing the boundary condition in (15), which comes from the von Neumann theory of the self-adjoint extensions. Our results are dependent on the self-adjoint extension parameter $\nu$. For the special value of $v=\infty$ we recover the results of [34]. Our expression for the scattering amplitude has an energy dependency. So, the helicity is not conserved in the scattering process. Last but not least, examining the poles of the $S$-matrix, an expression for the bound state energy was determined. The presence of bound states has not been discussed before.

\section{Competing Interests}

The authors declare that there is no conflict of interests regarding the publication of this paper.

\section{Acknowledgments}

Fabiano M. Andrade thanks Simone Severini and Sougato Bose by their hospitality at University College London. This work was partially supported by the CNPq, Brazil, Grants nos. 482015/2013-6 (Universal), 476267/2013-7 (Universal), 460404/2014-8 (Universal), 306068/2013-3 (PQ), and 311699/2014-6 (PQ), FAPEMA, Brazil, Grant no. 01852/14 (PRONEM), and FAPEMIG.

\section{References}

[1] L. Dolan and R. Jackiw, "Symmetry behavior at finite temperature," Physical Review D, vol. 9, no. 12, pp. 3320-3341, 1974.

[2] S. Weinberg, "Gauge and global symmetries at high temperature," Physical Review D, vol. 9, no. 12, pp. 3357-3378, 1974.

[3] A. D. Linde, "Phase transitions in gauge theories and cosmology," Reports on Progress in Physics, vol. 42, no. 3, pp. 389-437, 1979.
[4] T. W. Kibble, “Topology of cosmic domains and strings," Journal of Physics A: Mathematical and General, vol. 9, no. 8, pp. 13871398, 1976.

[5] J. Silk and A. Vilenkin, "Cosmic strings and galaxy formation," Physical Review Letters, vol. 53, no. 17, pp. 1700-1703, 1984.

[6] N. Turok and R. H. Brandenberger, "Cosmic strings and the formation of galaxies and clusters of galaxies," Physical Review D, vol. 33, no. 8, pp. 2175-2181, 1986.

[7] M. V. Manías, C. M. Naón, F. A. Schaposnik, and M. Trobo, "Non-abelian charged vortices as cosmic strings," Physics Letters $B$, vol. 171, no. 2-3, pp. 199-202, 1986.

[8] J. S. Dowker, "A gravitational Aharonov-Bohm effect," Il Nuovo Cimento B Series 10, vol. 52, no. 1, pp. 129-135, 1967.

[9] L. H. Ford and A. Vilenkin, "A gravitational analogue of the Aharonov-Bohm effect," Journal of Physics. A. Mathematical and General, vol. 14, no. 9, pp. 2353-2357, 1981.

[10] J. Stachel, "Globally stationary but locally static space-times: a gravitational analog of the Aharonov-Bohm effect," Physical Review D, vol. 26, no. 6, pp. 1281-1290, 1982.

[11] V. B. Bezerra, "Gravitational analogue of the Aharonov-Bohm effect in four and three dimensions," Physical Review D, vol. 35, no. 6, pp. 2031-2033, 1987.

[12] A. Aliev and D. Gal'tsov, "Gravitational Aharonov-Bohm radiation in string-generated conical space-time," Annals of Physics, vol. 193, no. 1, pp. 142-165, 1989.

[13] A. de Pádua Santos and E. R. Bezerra de Mello, "Non-Abelian cosmic strings in de Sitter and anti-de Sitter space," Physical Review D, vol. 94, no. 6, Article ID 063524, 2016.

[14] H. Hassanabadi and M. Hosseinpour, "Thermodynamic properties of neutral particle in the presence of topological defects in magnetic cosmic string background," The European Physical Journal C, vol. 76, no. 10, article no. 553, 2016.

[15] H. Weigel, M. Quandt, and N. Graham, "Isospin invariance and the vacuum polarization energy of cosmic strings," Physical Review D, vol. 94, no. 4, Article ID 045015, 2016.

[16] K. Jusufi, "Light deflection with torsion effects caused by a spinning cosmic string," European Physical Journal C, vol. 76, no. 6, article no. 332, 2016.

[17] J. P. Morais Graça, "Abelian cosmic string in the Starobinsky model of gravity," Classical and Quantum Gravity, vol. 33, no. 5, Article ID 055004, 2016.

[18] H. S. Vieira, V. B. Bezerra, and G. V. Silva, "Analytic solutions in the dyon black hole with a cosmic string: scalar fields, Hawking radiation and energy flux," Annals of Physics, vol. 362, pp. 576592, 2015.

[19] H. Cai, H. Yu, and W. Zhou, "Spontaneous excitation of a static atom in a thermal bath in cosmic string spacetime," Physical Review D, vol. 92, no. 8, Article ID 084062, 2015.

[20] K. Jusufi, "Hawking radiation via tunneling from the spacetime of a spinning cosmic string black holes," General Relativity and Gravitation, vol. 47, article no. 124, 2015.

[21] A. de Pádua Santos and E. R. Bezerra de Mello, "Gravitating non-abelian cosmic strings," Classical and Quantum Gravity, vol. 32, no. 15, Article ID 155001, 2015.

[22] L. B. Castro, "Quantum dynamics of scalar bosons in a cosmic string background," The European Physical Journal C, vol. 75, no. 6, article no. 287, 2015.

[23] A. Beresnyak, "The flow around a cosmic string. I. Hydrodynamic solution," Astrophysical Journal, vol. 804, no. 2, article no. 121, 2015. 
[24] H. Hassanabadi, A. Afshardoost, and S. Zarrinkamar, "On the motion of a quantum particle in the spinning cosmic string space-time," Annals of Physics, vol. 356, pp. 346-351, 2015.

[25] F. Niedermann, R. Schneider, S. Hofmann, and J. Khoury, "Universe as a cosmic string," Physical Review D, vol. 91, no. 2, Article ID 024002, 2015.

[26] H. F. S. Mota and M. Hindmarsh, "Big-bang nucleosynthesis and gamma-ray constraints on cosmic strings with a large Higgs condensate," Physical Review D, vol. 91, no. 4, Article ID 043001, 2015.

[27] J.-H. Wang, K. Ma, and K. Li, "Influences of a topological defect on the spin Hall effect," Physical Review A, vol. 87, no. 3, Article ID 032107, 2013.

[28] D. Chowdhury and B. Basu, "Effect of a cosmic string on spin dynamics," Physical Review D, vol. 90, no. 12, Article ID 125014, 2014.

[29] J. Carvalho, C. Furtado, and F. Moraes, "Dirac oscillator interacting with a topological defect," Physical Review A, vol. 84, no. 3, Article ID 032109, 2011.

[30] F. M. Andrade and E. O. Silva, "Effects of spin on the dynamics of the 2D Dirac oscillator in the magnetic cosmic string background," The European Physical Journal C, vol. 74, no. 12, article no. 3187, 2014.

[31] H. F. Mota and K. Bakke, "Noninertial effects on the ground state energy of a massive scalar field in the cosmic string spacetime," Physical Review D, vol. 89, no. 2, Article ID 027702, 2014.

[32] C. R. Muniz, V. B. Bezerra, and M. S. Cunha, "Landau quantization in the spinning cosmic string spacetime," Annals of Physics, vol. 350, pp. 105-111, 2014.

[33] J. Muñoz-Castañeda and M. Bordag, "Quantum vacuum interaction between two cosmic strings revisited," Physical Review D, vol. 89, no. 6, Article ID 065034, 2014.

[34] E. R. Bezerra de Mello, "Effects of anomalous magnetic moment in the quantum motion of neutral particle in magnetic and electric fields produced by a linear source in a conical spacetime," Journal of High Energy Physics, vol. 2004, no. 6, article 016, 2004.

[35] Y. Aharonov and A. Casher, "Topological quantum effects for neutral particles," Physical Review Letters, vol. 53, no. 4, pp. 319321, 1984.

[36] Y. Aharonov and D. Bohm, "Significance of electromagnetic potentials in the quantum theory," Physical Review, vol. 115, no. 3, pp. 485-491, 1959.

[37] W. Bulla and F. Gesztesy, "Deficiency indices and singular boundary conditions in quantum mechanics," Journal of Mathematical Physics, vol. 26, no. 10, pp. 2520-2528, 1985.

[38] S. Albeverio, F. Gesztesy, R. Hoegh-Krohn, and H. Holden, Solvable Models in Quantum Mechanics, AMS Chelsea Publishing, Providence, RI, Providence, RI, USA, 2nd edition, 2004.

[39] D. D. Sokolov and A. A. Starobinski, "On the structure of the curvature tensor at conical singularities," Soviet Physics, Doklady, vol. 22, p. 312, 1977.

[40] K. Bakke, J. R. Nascimento, and C. Furtado, "Geometric phase for a neutral particle in the presence of a topological defect," Physical Review D, vol. 78, Article ID 064012, 2008.

[41] I. Lawrie, A Unified Grand Tour of Theoretical Physics, Taylor \& Francis, Abingdon, UK, 3rd edition, 2012.

[42] R. H. Brandenberger, A.-C. Davis, and A. M. Matheson, "Callan-rubakov effect for strings," Nuclear Physics B, vol. 307, no. 4, pp. 909-923, 1988.
[43] M. G. Alford, J. March-Russell, and F. Wilczek, "Enhanced baryon number violation due to cosmic strings," Nuclear Physics B, vol. 328, no. 1, pp. 140-158, 1989.

[44] C. R. Hagen, "Aharonov-Bohm scattering of particles with spin," Physical Review Letters, vol. 64, no. 5, pp. 503-506, 1990.

[45] V. R. Khalilov, "Bound states of massive fermions in AharonovBohm-like fields," The European Physical Journal C, vol. 74, no. 1, article 2708, 2014.

[46] Ph. de Sousa Gerbert, "Fermions in an Aharonov-Bohm field and cosmic strings," Physical Review D, vol. 40, no. 4, pp. 13461349, 1989.

[47] V. R. Khalilov, "Creation of planar charged fermions in Coulomb and Aharonov-Bohm potentials," The European Physical Journal C, vol. 73, no. 8, article 2548, 2013.

[48] V. R. Khalilov and I. V. Mamsurov, "Scattering of a neutral fermion with anomalous magnetic moment by a charged straight thin thread," Theoretical and Mathematical Physics, vol. 161, no. 2, pp. 1503-1512, 2009.

[49] D. K. Park and J. G. Oh, "Self-adjoint extension approach to the spin-1/2 Aharonov-Bohm-Coulomb problem," Physical Review $D$, vol. 50, no. 12, p. 7715, 1994.

[50] D. K. Park, "Green's-function approach to two- and three-dimensional delta-function potentials and application to the spin-1/2 Aharonov-Bohm problem," Journal of Mathematical Physics, vol. 36, no. 10, p. 5453, 1995.

[51] R. Jackiw, Diverse Topics in Theoretical and Mathematical Physics, Advanced Series in Mathematical Physics, World Scientific, Singapore, 1995.

[52] M. Reed and B. Simon, Methods of Modern Mathematical Physics. II. Fourier Analysis, Self-Adjointness, Academic Press, New York, NY, USA, 1975.

[53] F. Gesztesy, S. Albeverio, R. Hoegh-Krohn, and H. Holden, "Point interactions in two dimensions: basic properties, approximations and applications to solid state physics," Journal für die Reine und Angewandte Mathematik, vol. 380, pp. 87-107, 1987.

[54] C. R. Hagen, "Comment on 'relativistic Aharonov-Bohm effect in the presence of planar Coulomb potentials"' Physical Review A, vol. 77, Article ID 036101, 2008.

[55] C. R. Hagen, "Aharonov-Bohm scattering amplitude," Physical Review D, vol. 41, no. 6, pp. 2015-2017, 1990.

[56] E. O. Silva, F. M. Andrade, C. Filgueiras, and H. Belich, "On Aharonov-Casher bound states," European Physical Journal C, vol. 73, no. 4, article no. 2402, 2013.

[57] A. S. Goldhaber, "Dirac particle in a magnetic field: symmetries and their breaking by monopole singularities," Physical Review $D$, vol. 16, no. 6, pp. 1815-1827, 1977. 

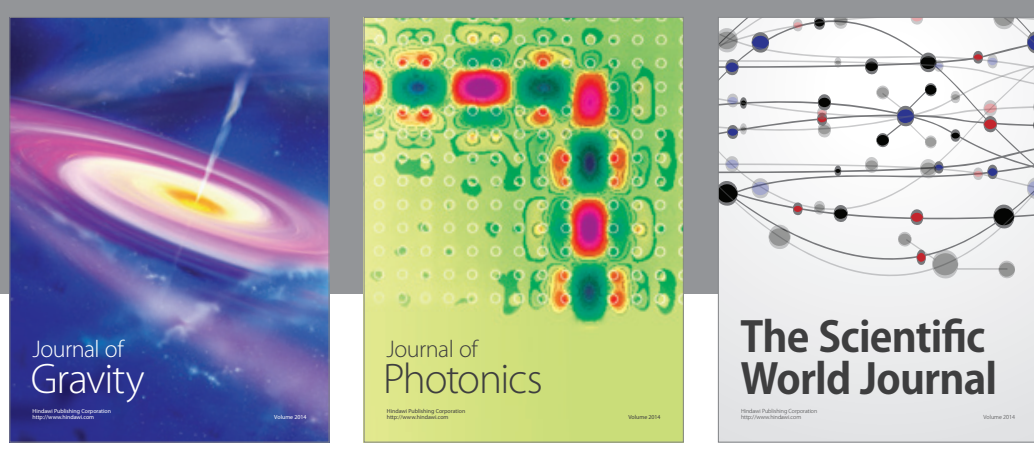

The Scientific World Journal
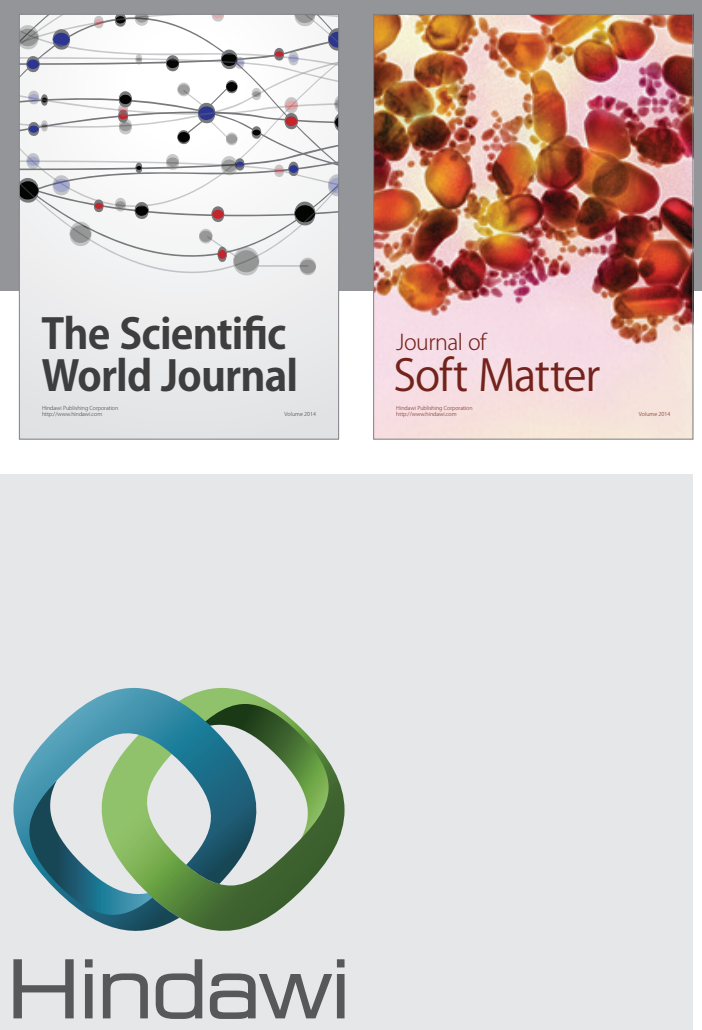

Submit your manuscripts at

https://www.hindawi.com
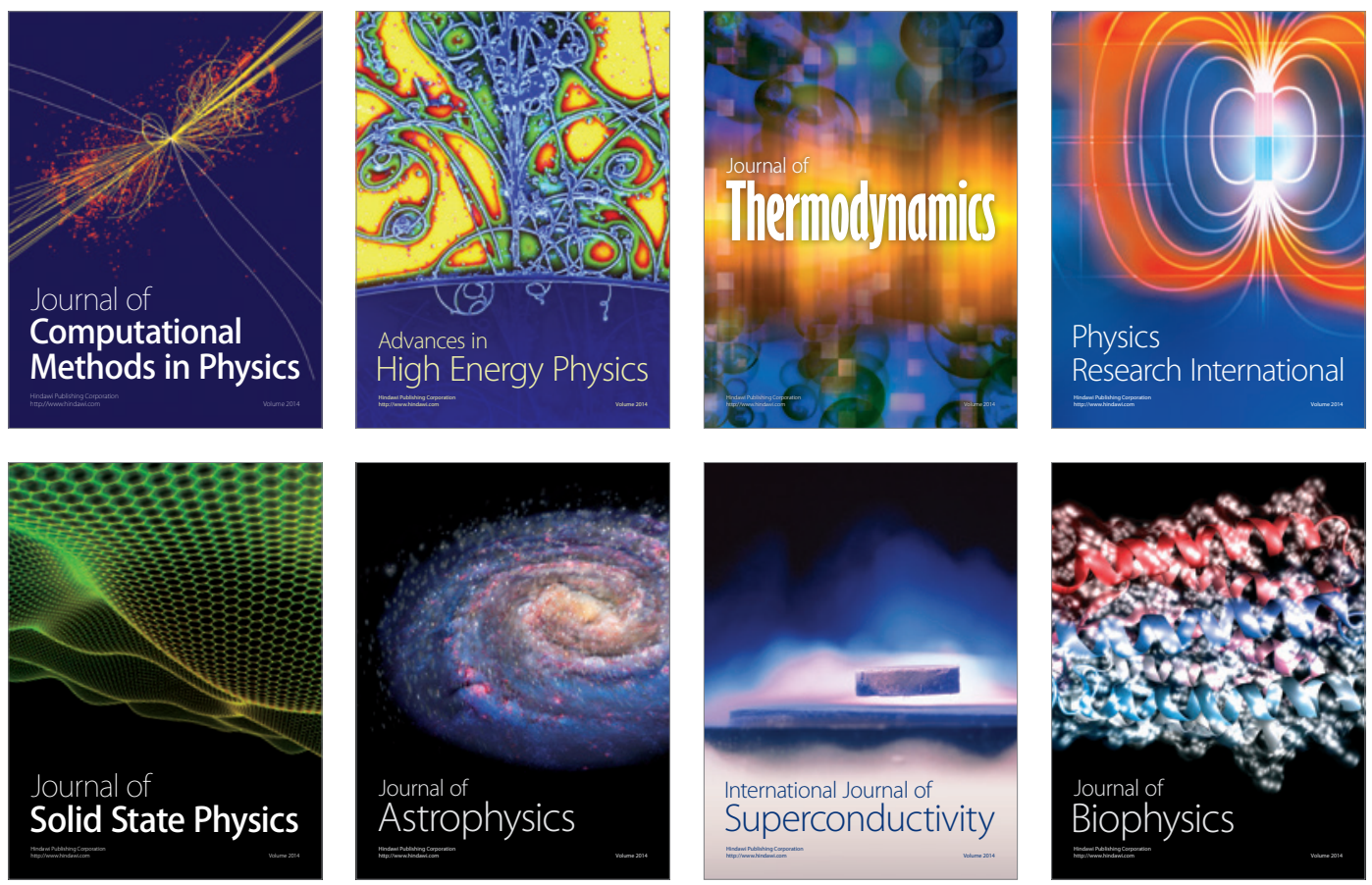
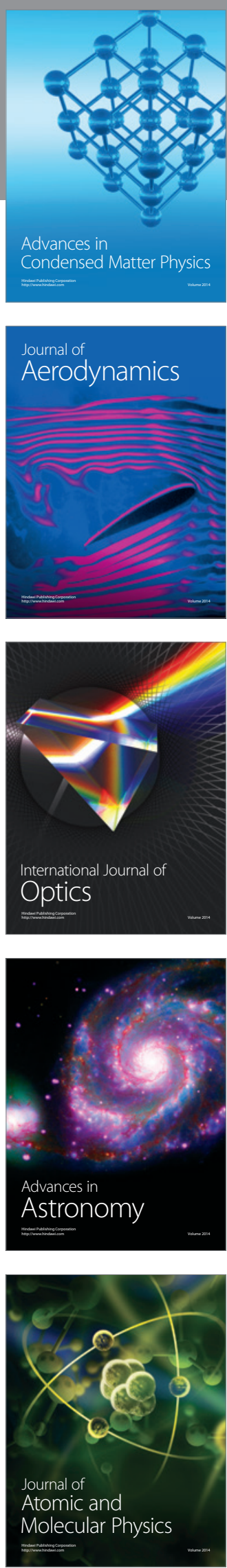